\title{
Wintersturmschäden im Schweizer Wald von 1865 bis 2014
}

Tilo Usbeck Eidgenössische Forschungsanstalt für Wald, Schnee und Landschaft WSL und Université de Neuchâtel (CH)*

\author{
Wintersturmschäden im Schweizer Wald von 1865 bis 2014
}

\begin{abstract}
Winterstürme verursachen die schwersten Schäden am Schweizer Wald. Für den vorliegenden Beitrag wurde untersucht, wie sich diese Sturmschäden in den Wäldern landesweit und in den einzelnen Kantonen seit 1865 bis 2014 in Abhängigkeit der Windstärke, des stehenden Holzvorrats und in Beziehung zur Waldfläche entwickelt haben. Im Studienzeitraum traten 26 Winterstürme mit je mindestens $70000 \mathrm{~m}^{3}$ Schadholz auf. Die Sturmschäden zeigen eine hohe Variabilität, sowohl absolut (Volumen in $\left.\mathrm{m}^{3}\right)$ als auch relativ zur Waldfläche $\left(\mathrm{m}^{3} / \mathrm{ha}\right)$ und zum stehenden Vorrat (\%). In den vergangenen 150 Jahren waren im Durchschnitt die Kantone Nidwalden, Freiburg, Aargau, Zürich und Zug am stärksten betroffen, mit Schäden von $2.2 \mathrm{~m}^{3} / \mathrm{ha}$ (Zürich) bis $3.1 \mathrm{~m}^{3} / \mathrm{ha}$ (Nidwalden) pro Ereignis. Um die Jahrtausendwende waren nicht nur die grössten Schäden zu verzeichnen, sondern es wurden auch die höchsten stehenden Vorräte und die höchsten Spitzenböen registriert.
\end{abstract}

Keywords: winter storm damage risk, windthrow, gust wind speed, spatial damage pattern, forest history doi: $10.3188 /$ szf.2015.0184

* Zürcherstrasse 111, CH-8903 Birmensdorf, E-Mail tilo.usbeck@wsl.ch

S türme entstehen als Folge von grossen Luftdruckgradienten unter Beteiligung grosser Temperaturunterschiede zwischen Warm- und nachfolgender Kaltluft. Erreichen die Winde Geschwindigkeiten von mehr als $32.7 \mathrm{~m} / \mathrm{s}(117.7 \mathrm{~km} / \mathrm{h}$; Stärke 12 auf der Beaufort-Skala), werden sie Orkane genannt. Seit 1954 werden Tief- und Hochdruckgebilde durch Namen bezeichnet, doch erst seit den Orkantiefs Vivian und Wiebke (1990) wurden die Medien auf die Namensgebung durch das Institut für Meteorologie der Fachuniversität Berlin aufmerksam. Extratropische Stürme treten in den Wintermonaten auf (Blizzards, europäische Winterstürme), da dann die Temperaturunterschiede in der Stratosphäre zwischen der Polarzone und dem Süden besonders gross sind und damit mehr kinetische Energie vorhanden ist. Winterstürme in Europa sind von West nach Ost verlaufende Zyklonbewegungen, die zum Wettergeschehen der Westwindzone gehören (Mitchell 2013). Trotz grosser Variabilität der Sturmaktivität wird für die Zukunft eine Zunahme von Winterstürmen in Europa erwartet (Donat et al 2011a, Donat et al 2011b). Demgegenüber steht allerdings die Feststellung, dass bei zunehmend höheren globalen Temperaturen die Temperaturunterschiede in der Stra- tosphäre abnehmen werden und damit auch die Häufigkeit der Stürme (Bengtsson et al 2009). Demnach müssten diese Stürme extremer sein bei kälterem Klima. Die Überlegungen zeigen, dass $\mathrm{Zu}$ sammenhänge oder direkte Verknüpfungen zum Klimawandel als Ursachen für die Entwicklung von Winterstürmen als eher unsicher befunden werden (Booth et al 2013, Burningham \& French 2013).

Wie auch immer, Winterstürme sind die grösste Naturgefahr für den Schweizer Wald. Sie können infolge ihrer grossen Ausdehnung in mehreren Regionen gleichzeitig Schaden anrichten (WSL \& BUWAL 2001). Solche Ereignisse ziehen oft auch Sekundärschäden mit grossen Mengen Käferholz nach sich (Forster \& Meier 2008).

Die Wirkung von Naturgefahren auf den Wald war schon vor dem Aufbau wissenschaftlicher Einrichtungen Gegenstand dauerhaften Monitorings. So wurden von vielen kantonalen Verwaltungen bereits kurz nach Mitte des 19. Jahrhunderts Informationen zu Waldschäden erhoben und in ihren Jahresberichten publiziert.

Für den vorliegenden Beitrag wurde untersucht, wie sich die von Winterstürmen verursachten Waldschäden in der Schweiz und in den einzelnen 
Kantonen seit 1865 in Abhängigkeit der Windstärke, des stehenden Holzvorrats und in Beziehung zur Waldfläche entwickelt haben.

\section{Material und Methoden}

\section{Eingrenzung der Aufgabenstellung}

Zunächst wurden quantitative und qualitative Informationen zu Sturmschäden von kommunalem bis nationalem Niveau gesammelt. Nach einer ersten Sichtung wurde die Aufgabenstellung präzisiert. Demnach wurden für die vorliegende Arbeit nur Daten berücksichtigt, die folgende Bedingungen erfüllten:

1) Zeithorizont: Winter 1865 bis Winter 2014 (Winter x: 1. Oktober des Jahres x-1 bis 31. März des Jahres $\mathrm{x}$ ),

2) räumliche Auflösung der Datensammlung: Kanton,

3) Schadenmerkmal: Volumen im Festmass,

4) Schaden-Untergrenze (kleinster «schwerer Schaden»): $70000 \mathrm{~m}^{3}$ schweizweit, wobei mindestens drei Kantone betroffen sein mussten. ${ }^{1}$

\section{Zielgrösse: Schadholzvolumen}

Als Datenquellen zur Ermittlung der Schadholzvolumen (Wurf- bzw. Bruchholz je Sturm respektive Sturmserie) dienten kantonale und eidgenössische Veröffentlichungen, insbesondere die kantonalen Rechenschaftsberichte von 1865 bis in die späten 1960er-Jahre. Seit den grossen Schäden der Winterstürme des Jahres 1967 wurde diese Datenquelle allerdings lückig bezüglich der Meldung kleinerer Schadenereignisse. Insbesondere lieferte sie seit den frühen 1970er-Jahren nur noch Infor-

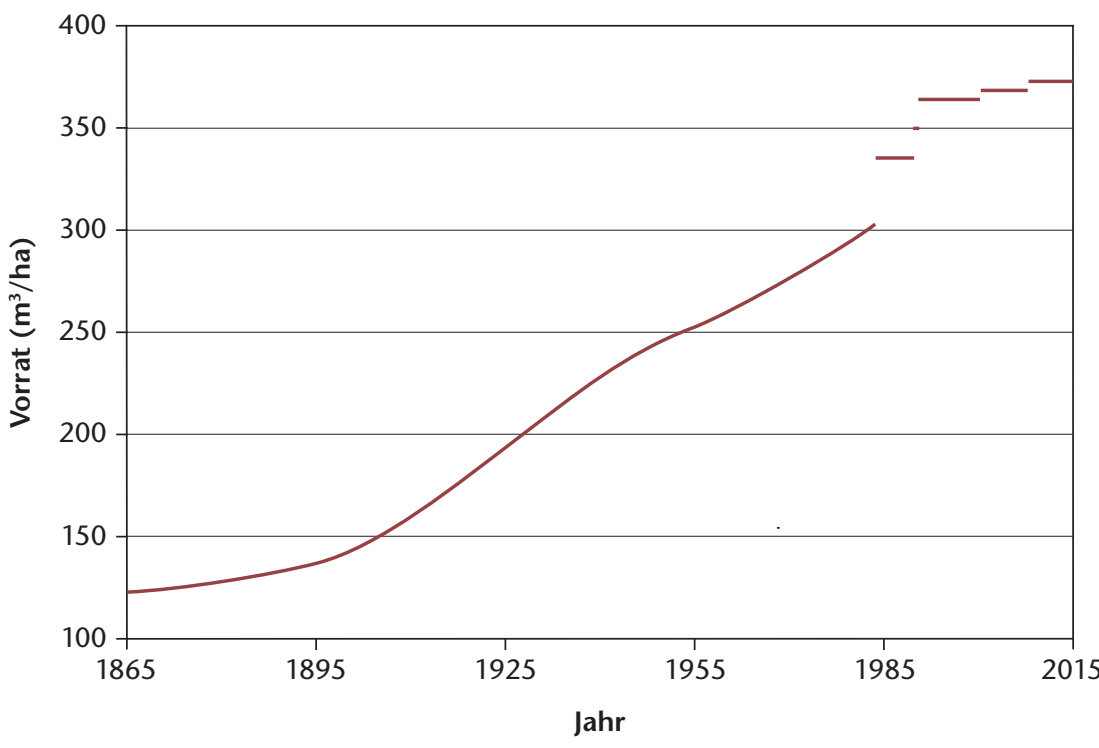

Abb 1 Entwicklung der Bezugsgrösse Vorrat pro Hektare. 1865 bis 1982: hypothetischer Vorrat gemäss Kurz et al (1998), ab 1983 Vorrat (der lebenden Bäume) pro Hektare gemäss Landesforstinventar (Auswertungseinheit: gemeinsamer zugänglicher Wald ohne Gebüschwald LFI1-LFI4b). mationen zu schweren Sturmschäden (Pfister 1998). Seit circa 2005 werden kantonale Sturmschadendaten ereignisabhängig beim Bundesamt für Umwelt (BAFU) gesammelt. Aufgrund dieser Unsicherheit der Schadendaten bei kleineren Sturmereignissen wurde die Schadenuntergrenze für die Untersuchung auf $70000 \mathrm{~m}^{3}$ je Ereignis festgesetzt. Da das metrische System erst ab 1877 verbindlich verwendet wurde, mussten die in den frühen Quellen verwendeten Volumenangaben (Kubikfuss, Normalklafter) umgerechnet werden.

Die totalen Schadholzvolumen wurden als gesamtschweizerische und kantonale Werte aus den jeweiligen Publikationen übernommen. Wo die Totale nur auf kantonalem Niveau verfügbar waren, wurde der nationale Wert aus diesen aufsummiert. War das Schadholzvolumen in einem Kanton nur für eine bestimmte Eigentumskategorie erhoben worden, wurde es auf den entsprechenden Anteil an der Waldfläche des jeweiligen Kantons bezogen.

\section{Bezugsgrössen: Waldfläche, Vorrat und Windstärke}

Die Schadholzvolumen wurden in Beziehung gesetzt zum Vorrat (=Vorrat der lebenden Bäume; Schadanteil) und zur Waldfläche (relativer Schaden). Die früheste Erhebung der Waldflächen aller Kantone stammt zwar aus den Jahren 1858 bis 1860 (Landolt 1862), und seit 1877 werden die Waldflächen aller Kantone für die Forststatistik ermittelt. Da aber die Erhebungsmethoden sowie die Walddefinitionen früherer Erhebungen weitgehend unbekannt sind (Brändli 2000), wurden hier ausschliesslich die Daten des LFI (EAFV 1988, Brassel \& Brändli 1999, Brändli 2010, Abegg et al 2014) verwendet.

Der Vorrat ist einer der grundlegenden forstlichen Parameter. Für die Schweiz sind aber nur wenige grossflächige Vorratsermittlungen bekannt, und nur jene des LFI betreffen den gesamten Wald. Für die Untersuchung wurde daher auf die Arbeit von Kurz et al (1998) zurückgegriffen, die den landesweit durchschnittlichen Vorrat für den Zeitraum von 1800 bis 2050 abgeschätzt haben. Konkret wurden die Daten von Kurz et al (1998) bis zum Wirksamwerden des LFI (1983) verwendet, ab da die LFIDaten für den stehenden Holzvorrat (Abbildung 1).

Als Winddaten wurden die Spitzenböen der Messungen der Station Zürich von MeteoSchweiz verwendet, da diese eine lange Reihe repräsentativer Daten zu Winterstürmen in Höhenlagen unterhalb der Waldgrenze bietet (Usbeck et al 2010a). Ab 1872 wurden die (maximalen) Windgeschwindigkeiten mittels Anemometer gemessen und aufgezeichnet, ab 1891 sind die Winddaten homologisiert. Aus den maximalen Spitzenböen je Sturmereignis wurden

\footnotetext{
1 Bei einem Vorrat von $350 \mathrm{~m}^{3} /$ ha entsprechen $70000 \mathrm{~m}^{3}$ einem Totalschaden auf einer Fläche von 200 ha.
} 
die Winddrücke ermittelt. Aus den Winddrücken und Schadholzvolumen respektive Schadanteilen wurde der Pearson-Korrelationskoeffizient (r) berechnet und mittels linearen Modells (lm) die Signifikanz auf dem 5\%-Niveau getestet, sowohl für Schweizer als auch für die Zürcher Daten.

\section{Datenqualität}

Die Schadholzvolumen wurden und werden auf kantonaler Ebene und ohne schweizweit einheitliche Methodik erhoben. Es fehlen somit Gütemerkmale dieser Daten für den interkantonalen Vergleich. Die Daten des LFI zu Waldfläche und Vorrat sind methodisch abgesichert. Allerdings sind diese Daten

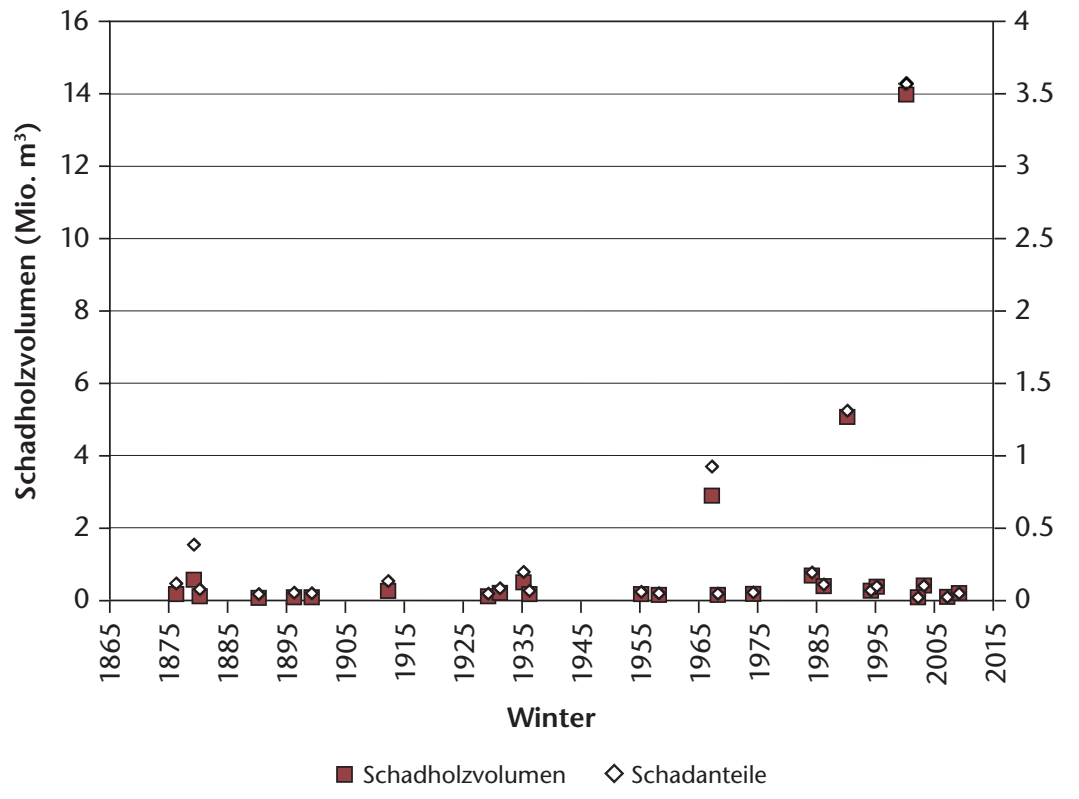

Abb 2 Schadholzvolumen und Schadanteile der 26 schweren Winterstürme (Schaden $\geq 70000 \mathrm{~m}^{3}$ je Ereignis) in der Schweiz von 1865 bis 2014.

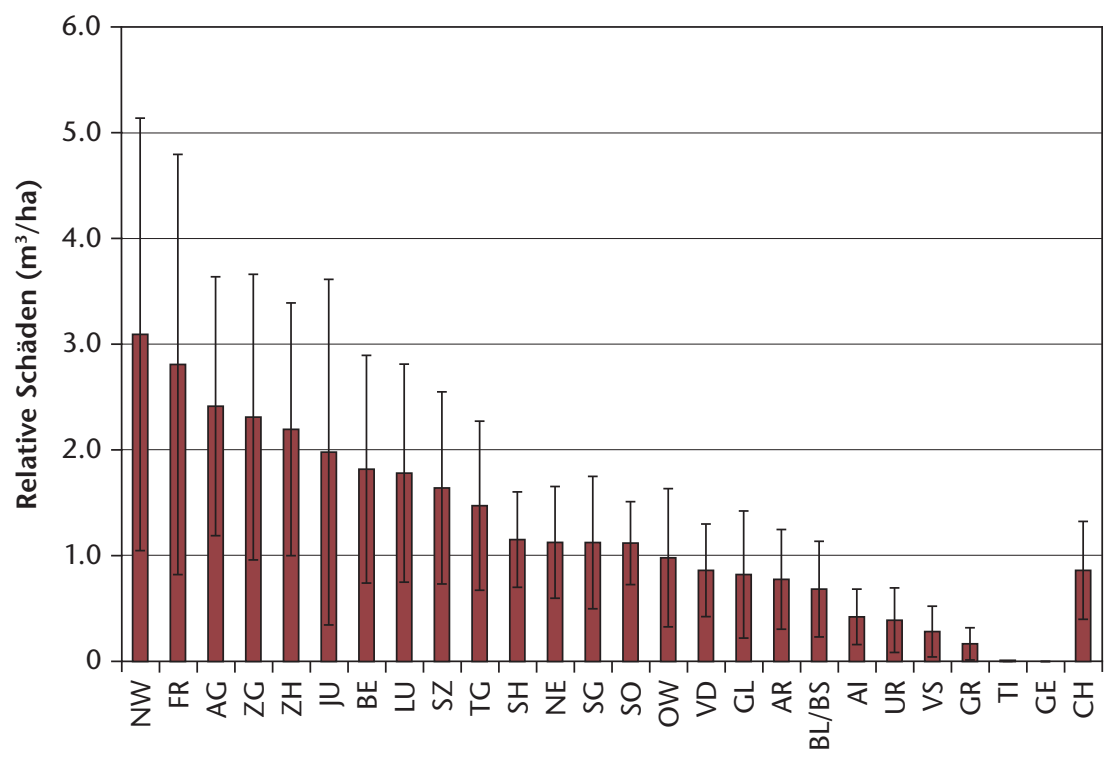

Kantone resp. Schweiz

Abb 3 Relative Schäden (Schadholzvolumen pro Hektare Waldfläche) pro Kanton und für die Schweiz. Angegeben sind der Mittelwert und die Standardabweichung der 26 schweren Winterstürme (Schaden $\geq 70000 \mathrm{~m}^{3}$ je Ereignis) von 1865 bis 2014 in der Schweiz. erst ab dem Jahr 1983 verfügbar. Für die Jahre 1865 bis 1982 mussten Annäherungen gesucht werden. So wird für die Waldausdehnung früherer Jahre auch die Waldfläche gemäss LFI angenommen. Für den Vorrat wird der hypothetische Vorrat von Kurz et al (1998) verwendet, der mittels Metadaten hergeleitet wurde, ohne bestimmte Genauigkeitsmasse zu beanspruchen.

Die Winddaten werden seit 1980 innerhalb eines Messnetzes erhoben (ANETZ, jetzt SwissMetNet) und unterliegen den Qualitätsanforderungen der MeteoSchweiz. Zu allen früheren Daten fehlen entsprechende Qualitätsangaben.

\section{Resultate}

Anzahl Winterstürme und Schadholzmengen

Von 1865 bis 2014 wurden in der Schweiz 26 schwere Winterstürme mit mindestens $70000 \mathrm{~m}^{3}$ Schadholz registriert (Abbildung 2). Für diese wurden in $84.1 \%$ der gesamthaft 634 Schadenereignisse (Summe aller kantonalen Schadenereignisse aller Stürme) Daten auf kantonalem Niveau gefunden. Für die Kantone Genf und Tessin fehlen in der Regel Schadenangaben. Die am lückenhaftesten dokumentierten Sturmschäden betreffen jene der Winter 1968 und 2002, welche jeweils den katastrophalen Ereignissen 1967 und 1999 in kurzem Abstand folgten.

23 der 26 Winterstürme haben Schadholzmengen von weniger als 2 Mio. $\mathrm{m}^{3}$ verursacht, und nur drei liegen über dieser Zahl. Diese drei Stürme hinterliessen Schäden von circa 2.9 Mio. m³ (1967), 5.1 Mio. $\mathrm{m}^{3}$ (Vivian vom Februar 1990; Summe aller kantonalen Schadenmeldungen) und 14 Mio. $\mathrm{m}^{3}$ (Lothar vom Dezember 1999). Dabei ist der grösste beobachtete Schaden (Lothar) 20-mal so gross wie die untere Erfassungsgrenze für diese Studie.

Eine grosse Variabilität zeigt sich auch in Bezug auf die Schadanteile, die oft weniger als $0.1 \%$ des Vorrats betragen, aber auch einen Wert von 3.6\% erreichen können. Treten über den Studienzeitraum ähnlich grosse Schadholzmengen auf, so werden mit fortschreitender Zeit die Schadanteile geringer, was hauptsächlich durch den zunehmenden stehenden Vorrat verursacht wird.

Die Winterstürme der Jahre 1967, 1990 und 1999 verursachten nicht nur extrem grosse Schadholzmengen, sondern auch die höchsten Schadanteile. Sie zerstörten 0.9\% (1967), 1.3\% (1990; Vivian) und 3.6\% des Holzvorrates (1999; Lothar). Die Schäden im Zuge dieser drei Stürme können nicht allein mit der Zunahme des Vorrats im Untersuchungszeitraum erklärt werden (Abbildung 2).

\section{Schäden je Kanton}

Die Mittelwerte der Schadholzvolumen je Hektar Waldfläche zeigen mit Werten von 0.0 bis $3.1 \mathrm{~m}^{3} /$ 


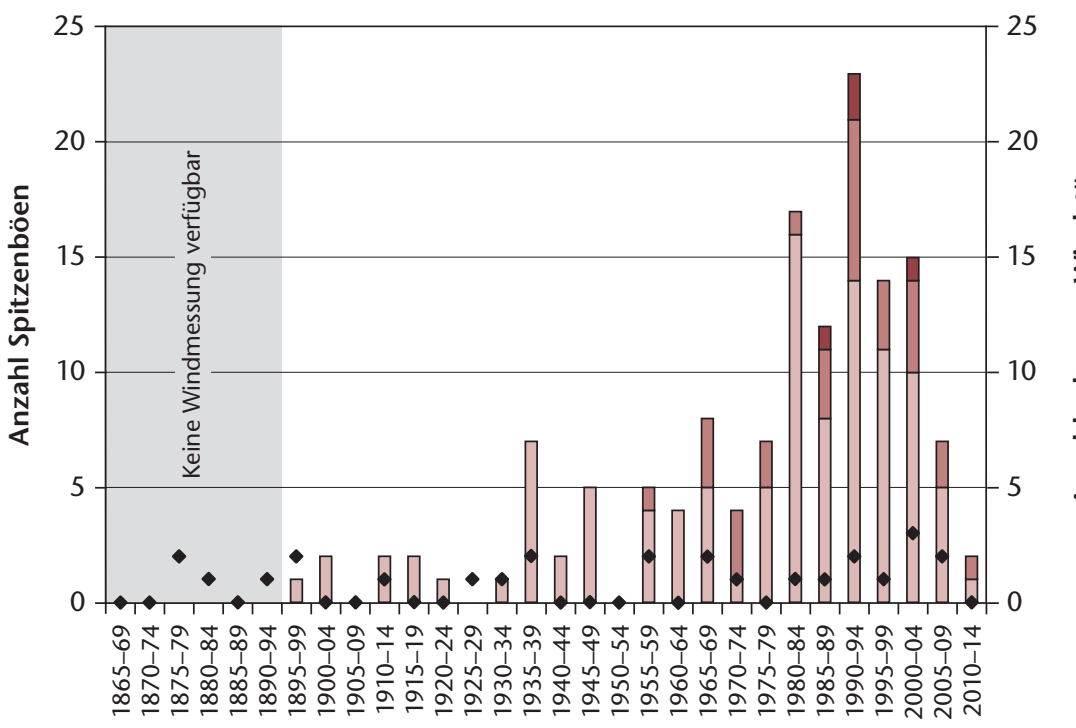

5-Winter-Intervall

Spitzenböen: $\square 30.0-<35.0 \mathrm{~m} / \mathrm{s} \square 35.0-<40.0 \mathrm{~m} / \mathrm{s} \square>40.0 \mathrm{~m} / \mathrm{s} \bullet$ Schwerer Windsturm

Abb 4 Anzahl täglicher Spitzenböen $\geq 30.0 \mathrm{~m} / \mathrm{s}$ an der SwissMetNet-Station Zürich-Fluntern (ab Periode 1895-1899) und Anzahl schwerer Winterstürme (Schaden $\geq 70000 \mathrm{~m}^{3}$ je Ereignis; ab Periode 1865-1869) je 5-Winter-Intervall.

ha grosse Unterschiede zwischen den Kantonen (Abbildung 3). Besonders hohe Werte sind in den Kantonen Nidwalden, Freiburg, Aargau, Zug und Zürich zu finden. Dagegen wurden sehr geringe Werte für die Kantone Genf, Tessin und Graubünden ermittelt.

\section{Windstärke und Schäden}

Spitzenböen mit mehr als $30 \mathrm{~m} / \mathrm{s}(108 \mathrm{~km} / \mathrm{h})$ wurden in Zürich am häufigsten zwischen 1980 und 2004 gemessen (Abbildung 4). Kleinere Frequenzen betreffen die Jahre 1935 bis 1979 sowie die letzten zehn Jahre (2005-2014). Nur selten wurden Spitzenböen in den Jahren 1895 bis 1934 registriert. Der Zusammenhang zwischen Winddruck und Waldschaden im Kanton Zürich und in der gesamten Schweiz ist mit Korrelationskoeffizienten von $\mathrm{r}_{\mathrm{ZH}}=0.60$ und $\mathrm{r}_{\mathrm{CH}}=0.63$ jeweils signifikant $\left(\mathrm{p}_{\mathrm{ZH}}=0.009, \mathrm{p}_{\mathrm{CH}}=0.002\right)$. Dabei sind die Schadenmengen von Zürich mit jenen für die ganze Schweiz erstaunlich gut korreliert $(\mathrm{r}=0.97, \mathrm{p}<0.001)$

\section{Zeitliche Entwicklung der Wintersturm- schäden}

Im 5-Winter-Intervall 2000 (Winter 1999/2000) bis 2004 (Winter 2003/2004) zogen gleich drei schwere Winterstürme über den Schweizer Wald, was die grösste zeitliche Dichte an schweren Stürmen in den vergangenen 150 Jahren darstellt. In den übrigen 29 Intervallen wurden höchstens zwei derartige Ereignisse gezählt (Abbildung 4). Bezogen auf den Schadanteil am Vorrat (Abbildung 2), ergeben sich damit vier grosse Schadenereignisse in den letzten 150 Jahren: Winter 1879, 1967, 1990 (Vivian) und 2000 (Lothar).

\section{Diskussion}

\section{Datenqualität}

Ein zentraler Punkt in der vorliegenden Untersuchung ist die Qualität der Quellen, was sowohl die Ziel- als auch die Bezugsgrössen betrifft. Mit der gewählten Schwelle von mindestens $70000 \mathrm{~m}^{3}$ Schadholz pro Ereignis wurde ein Mass gewählt, welches über den ganzen Beobachtungszeitraum gesehen recht zuverlässige Angaben zum Schadholzvolumen geben dürfte. Insbesondere ist durch die Wahl einer hohen Untergrenze die Ungewissheit über die Dimension kleiner Schadenereignisse infolge fehlender Meldungen ausgeschaltet.

Dagegen sind die Angaben zu Schadanteilen und relativen Schäden mit grösseren Unsicherheiten behaftet, weil die Bezugsgrössen Vorrat und Waldfläche für die Zeit vor den Landesforstinventaren, d.h. von 1865 bis 1982, mit Metadaten modelliert (Vorrat) respektive mit den Werten des LFI1 (Waldfläche) angenähert wurden. Mit der getroffenen Annahme wird die Waldfläche vor 1983 deutlich überschätzt (Brändli 2000, Ginzler et al 2011) und damit der relative Schaden klar unterschätzt. Für die ältesten Sturmereignisse dürfte der relative Schaden entsprechend rund 30 bis $40 \%$ höher liegen.

Die Werte im Vorratsmodell von Kurz et al (1998) sind besonders für die frühen Jahre mit Vorsicht zu verwenden. So wurde als Startvorrat im Jahr 1800 ein Wert von 110 m³ $^{3}$ ha festgelegt, dies in Anlehnung an den Zustand skandinavischer Wälder vor 200 Jahren. Als nächster Referenzpunkt für das Kurz-Modell wurden die Werte $252 \mathrm{~m}^{3} / \mathrm{ha}$ (1952) und $278 \mathrm{~m}^{3} / \mathrm{ha}$ (1972) eingesetzt, den Publikationen von EIFJF (1954) und Ott (1972) folgend. Eine kritische Gegenüberstellung der Modellwerte nach Kurz et al (1998) mit gemessenen Vorräten vor 1952 ist nicht nur aufwendig, weil reale Werte mit grossem Aufwand recherchiert werden müssen, sie betrifft auch nur ausgesuchte Regionen oder Talschaften. Für 1925 geben Kurz et al (1998) zum Beispiel einen Wert von $202 \mathrm{~m}^{3}$ /ha an, der mit dem Wert von $188 \mathrm{~m}^{3} /$ ha bei Bürgi (1998) für das Zürcher Unterland und Weinland recht gut übereinstimmt. Dagegen eruierten Gimmi et al (2009) für das Alptal im selben Jahr $238 \mathrm{~m}^{3} /$ ha. Für die beiden Beispiele sind die Abweichungen der modellierten Vorratswerte erstaunlich gering.

Die Windmessreihe in Zürich kann als beste ihrer Art für die Schweiz betrachtet werden (Usbeck et al 2010a), und weltweit gibt es nur wenige ähnlich lange Reihen. Obwohl der Standort der Windmessanlage innerhalb von Zürich dreimal wechselte (vom ETH-Hauptgebäude zur MeteoSchweiz) und während der langen Zeitspanne vier verschiedene Messgeräte zum Einsatz kamen, konnte aufgrund des gleichbleibenden Messprinzips (Schalenanemometer) und vieler verfügbarer Metadaten eine Homogenisierung 


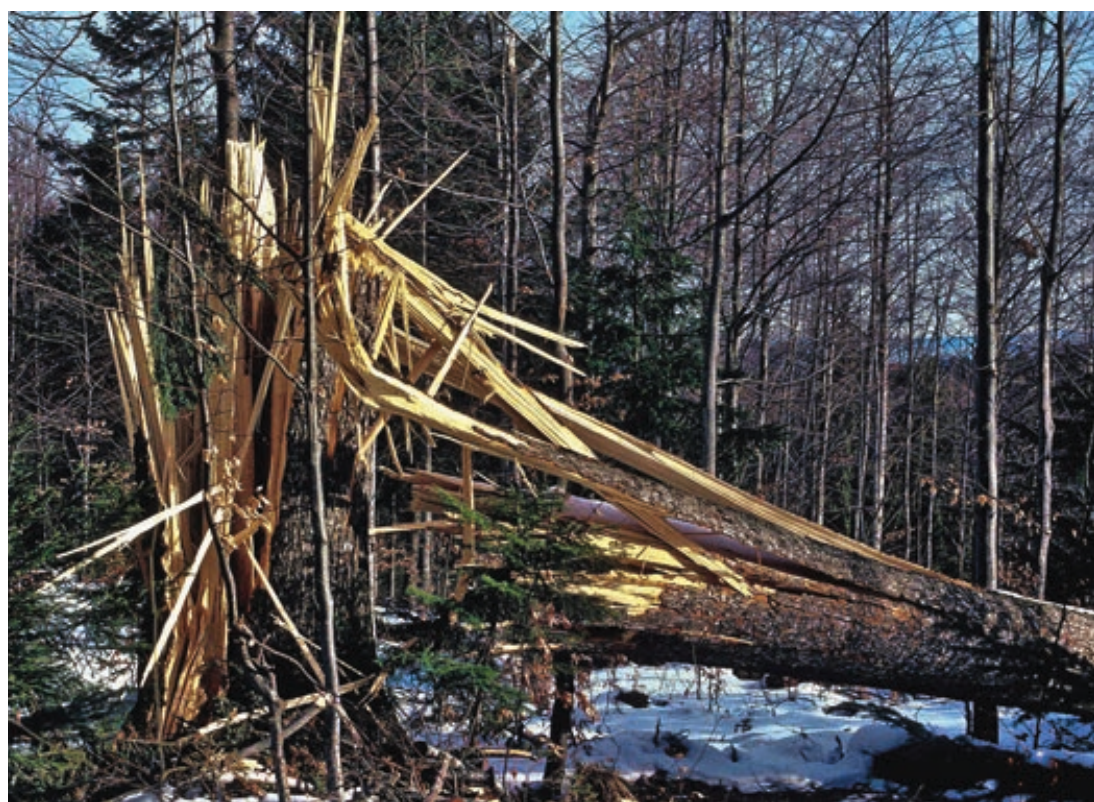

Abb 5 Ein in einem Wintersturm geknickter Einzelbaum. Foto: Heinz Jost

der Datenreihe vorgenommen werden (Usbeck et al 2010a). Die homogenisierten Daten gleichen für die Periode von 1969 bis 1999 auch den Messreihen von deutschen Städten (Münchner Rück 2010). Die wenigen Spitzenböen $\geq 30 \mathrm{~m} / \mathrm{s}$ in der ersten Hälfte der Untersuchungsperiode stehen auch im Einklang mit den festgestellten geringen Waldschäden durch Stürme.

\section{Risiko von Wintersturmschäden}

Winterstürme verursachten wiederholt Waldschäden, sowohl in der Schweiz als auch in Zentralund Nordeuropa (Schelhaas et al 2003). Die vorliegende Untersuchung legt die Vermutung nahe, dass in den letzten Jahrzehnten die Waldschäden durch Winterstürme deutlich zugenommen haben. Die 150-jährige Aufzeichnung kann aber auch dahingehend interpretiert werden, dass zwischen 1880 und 1967 kaum grössere Schäden zu verzeichnen waren. Auf eine solche Katastrophenlücke, die im Übrigen auch für Überschwemmungen in der Schweiz zu beobachten ist, weist Pfister $(1999,2009)$ wiederholt hin. Bereits in früheren Zeiten gab es offensichtlich lange Perioden ohne Wintersturmschäden (Landolt 1861).

Vor dem Hintergrund langer Zeitreihen lassen sich die besonders prägenden Windwurfereignisse Vivian und Lothar besser einordnen. Eine Verwendung der Daten für Prognosen muss aber sehr sorgfältig erfolgen. Beispielsweise hätte eine lineare Extrapolation der Spitzenböen über den Studienzeitraum hinaus Werte um $60 \mathrm{~m} / \mathrm{s}$ für die Wende vom 21. zum 22. Jahrhundert zur Folge, aber solche von lediglich $15 \mathrm{~m} / \mathrm{s}$ für die Wende vom 18. zum 19. Jahrhundert. Dem höheren Wert entsprechen die maximalen Böenspitzen, die während Lothar gemessen wurden. Dagegen erreichen Nordwinde (Bise) während der Winterzeit oft Stärken von 15 m/s.
Die grossräumliche Verteilung des Schadenrisikos dürfte hingegen auch ausserhalb des Studienzeitraums Gültigkeit besitzen. Angesichts der grossen Regelmässigkeit der Zyklonbewegungen von West nach Ost, welche unsere Winterstürme letztlich produzieren (Seidl et al 2014b), dürften auch in Zukunft vor allem das schweizerische Mittelland und die nördlichen Voralpen von grösseren Sturmschäden betroffen sein, mit abnehmender Schadintensität gegen Osten (WSL \& BUWAL 2001).

\section{Schadholzmengen}

Das Risiko für den Wald, von einem Wintersturm geschädigt zu werden, ist indes nicht nur vom Sturm, sondern auch von vielen anderen Faktoren abhängig (Hanewinkel et al 2015, dieses Heft), insbesondere von der Baumhöhe (Abbildung 5), mit der der stehende Vorrat stark korreliert ist. Am Anfang des Studienzeitraumes war dieses Risiko bei mittleren Vorräten von weniger als $150 \mathrm{~m}^{3} /$ ha wesentlich geringer als heute, wo die Wälder im Durchschnitt einen Vorrat von $374 \mathrm{~m}^{3} /$ ha erreichen (Abegg et al 2014). Sowohl die absoluten als auch die relativen Schadholzmengen haben in den vergangenen 150 Jahren stark zugenommen (Usbeck et al 2010b), ein Trend, der nicht nur in der Schweiz, sondern auch in ganz Europa feststellbar ist (Schelhaas et al 2003). Das Ausbleiben rekordhoher Sturmschäden in den vergangenen 15 Jahren hat demzufolge damit zu tun, dass die Schweiz nur marginal von weiteren grossen Winterstürmen getroffen wurde (z.B. 2007 Kyrill und 2009 Quinten). Geringere Holzvorräte würden das Schadenpotenzial bei ähnlich hohen Windstärken wie während Lothar wohl deutlich reduzieren.

Unter Berücksichtigung extremer Windgeschwindigkeiten der vergangenen 150 Jahre (Brönnimann et al 2012) und der Ergebnisse aktueller Modellrechnungen (Della-Marta \& Pinto 2009, Seidl et al 2014a) kann man davon ausgehen, dass Winterstürme im Schweizer Wald auch künftig schwere Schäden verursachen werden. Mechanische Belastungen, die aus Windgeschwindigkeiten von mehr als $40 \mathrm{~m} / \mathrm{s}$ resultieren, liegen Beobachtungen zufolge allgemein jenseits der Wurf- beziehungsweise Bruchresistenz (Bock et al 2004, Wohlgemuth et al 2008). Derartige Belastungen traten im Studienzeitraum auf, und sie dürften sich in der Zukunft wiederholen.

Die Entwicklung der Wintersturmschäden widerspiegelt sich auch in der gesellschaftlichen Wahrnehmung. So wurden der Sturmschaden vom 20. Februar 1879 (570979 m³) als eigentliche Katastrophe dargestellt (Coaz 1880), der Sturmschaden vom 23. Februar 1935 (über $500000 \mathrm{~m}^{3}$ ) als die Lage der Forstwirtschaft und des Holzhandels sowie die Nachhaltigkeit ungünstig beeinflussend (EIFJF 1936) und der Sturmschaden vom 27. November 1983 
$\left(550000 \mathrm{~m}^{3}\right)$ als $\mathrm{zu}$ grösseren Schwierigkeiten auf dem bereits überlasteten Nadelindustrieholzmarkt führend (Anonymus 1984). Die Schäden des Orkans Vivian im Jahre 1990 wurden schliesslich als die schwersten bezeichnet, die in der Schweiz bis dahin beobachtet wurden. Heute werden Schäden als von nationaler Bedeutung betrachtet, wenn sie sich im Bereich einer durchschnittlichen Jahresnutzung bewegen (BAFU 2008). Dieses Kriterium erfüllen gerade noch die Orkane Vivian und Lothar.

\section{Folgerungen}

Winterstürme sind in Europa wiederkehrende Ereignisse, die vom meteorologischen Standpunkt her als nichts Aussergewöhnliches zu betrachten sind. Dass die Schweiz in den letzten Jahren von schweren Wintersturmschäden verschont blieb, bedeutet nicht, dass die Windstärke der Stürme abgenommen hat. Vielmehr zogen die Sturmzentren zum überwiegenden Teil nördlich der Schweiz vorbei.

Durch die besondere Topografie der Schweiz werden die Inner- und Südalpen weniger von Winterstürmen heimgesucht. Mit Ausnahme von Vivian sind alle schweren Winterstürme von West nach Ost gezogen und von den nördlichen Voralpen am Eindringen in die inneren und südlichen Alpenteile gehindert worden, wobei das untere Rhonetal weniger stark geschützt war als beispielsweise die Täler des Vorder- und Hinterrheins. Vorratsreiche Wälder entlang des Alpennordhangs, im Jura und besonders im Mittelland dürfen dementsprechend als am stärksten windwurfgefährdet betrachtet werden.

Der Holzvorrat nimmt in vielen Regionen in der Schweiz weiterhin zu (Abegg et al 2014). Davon kann abgeleitet werden, dass das Schadholzvolumen durch Winterstürme, sofern sie diese Regionen treffen, nicht abnehmen, sondern zunehmen dürfte (Seidl et al 2014a). Eine Vorratsminderung, zum Beispiel durch kürzere Umtriebszeiten, dürfte sich auf die Schadholzmengen günstig auswirken, würde aber wohl anderen Interessen wie denjenigen des Natur- und Landschaftsschutzes zuwiderlaufen.

Eingereicht: 19. Dezember 2014, akzeptiert (mit Review): 14. April 2015

\section{Literatur}

ABEGG M, BRÄNDLI UB, CIOLDI F, FISCHER C, HEROLD-BONARDI A ET AL (2014) Viertes Schweizerisches Landesforstinventar - Ergebnistabellen und Karten im Internet zum LFI 2009-2013 (LFI4b). Birmensdorf: Eidgenöss Forsch.anst WSL. www.Ifi.ch/ resultate (4.3.2015).

ANONYMUS (1984) Abfluss von Nadelindustrieholz beschleunigen. Wald Holz 54: 399-400.

BAFU (2008) Sturmschaden-Handbuch. Vollzugshilfe für die Bewältigung von Sturmschadenereignissen von nationaler Bedeutung im Wald. Bern: Bundesamt Umwelt, Umwelt-Vollzug 0801. 241 p.
BENGTSSON L, HODGES KI, KEENLYSIDE N (2009) Will extratropical storms intensify in a warmer climate? J Clim 22:22762301.

BOCK J, VINKLER I, DUPLAT P, RENAUD JP (2004) Stabilité au vent des hêtraies: les enseignements de la tempête de 1999. Forêt Entreprise 156: 34-38.

BOOTH JF, WANG SG, POLVANI L (2013) Midlatitude storms in a moister world: lessons from idealized baroclinic life cycle experiments. Clim Dyn 41: 787-802.

BRÄNDLI UB (2000) Waldzunahme in der Schweiz - gestern und morgen. Birmensdorf: Eidgenöss Forsch.anstalt WSL, Inf.bl Forsch.bereich Landsch 45: 1-4.

BRÄNDLI UB (2010) Schweizerisches Landesforstinventar. Ergebnisse der dritten Erhebung 2004-2006. Birmensdorf: Eidgenöss Forsch.anstalt WSL. 312 p.

BRASSEL P, BRÄNDLI UB (1999) Schweizerisches Landesforstinventar. Ergebnisse der Zweitaufnahme 1993-1995. Bern: Haupt. 442 p.

BRÖNNIMANN S, MARTIUS O, VON WALDOW H, WELKER C, LUTERBACHER J ET AL (2012) Extreme winds at northern mid-latitudes since 1871. Meteorol Z 21: 1327.

BÜRGI M (1998) Waldentwicklung im 19. und 20. Jahrhundert. Veränderungen in der Nutzung und Bewirtschaftung des Waldes und seiner Eigenschaften als Habitat am Beispiel der öffentlichen Waldungen im Zürcher Unter- und Weinland. Beih Schweiz Z Forstwes 84: 1-234.

BURNINGHAM H, FRENCH J (2013) Is the NAO winter index a reliable proxy for wind climate and storminess in northwest Europe? Int J Climat 33: 2036-2049.

COAZ J (1880) Die Stürme vom 20. Februar, 25. Juni und 5. Dezember 1879 und der durch dieselben in den Waldungen der Schweiz verursachte Schaden. Bern: Jenet \& Reinert. 57 p.

DELLA-MARTA PM, PINTO JG (2009) Statistical uncertainty of changes in winter storms over the North Atlantic and Europe in an ensemble of transient climate simulations. Geophys Res Lett 36, L14703.

DONAT MG, LECKEBUSCH GC, WILD S, ULBRICH U (2011A) Future changes in European winter storm losses and extreme wind speeds inferred from GCM and RCM multi-model simulations. Nat Hazards Earth Syst Sci 11: 1351-1370.

DONAT MG, RENGGLI D, WILD S, ALEXANDER LV, LECKEBUSCH GC ET AL (2011B) Reanalysis suggests long-term upward trends in European storminess since 1871. Geophys Res Lett 38: 1-6.

EAFV (1988) Schweizerisches Landesforstinventar: Ergebnisse der Erstaufnahme 1982-1986. Birmensdorf: Eidgenöss Anst Forstl Vers.wes, Ber 305. 375 p.

EIFJF (1936) Schweizerische Forststatistik 1935. Bern: Eidgenössisches Statistisches Amt, Schweizerische Forststatistik 10: 1-24.

EIFJF (1954) Der stehende Holzvorrat in den öffentlichen Waldungen der Schweiz. Erhebungen 1951/52. Bern: Eidgenössische Inspektion für Forstwesen, Jagd und Fischerei. 34 p.

FORSTER B, MEIER F (2008) Sturm, Witterung und Borkenkäfer. Birmensdorf: Eidgenöss Forsch.anstalt WSL, Merkbl Prax 44. $8 \mathrm{p}$.

GIMMI U, WOLF A, BÜRGI M, SCHERSTJANOI M, BUGMANN H (2009) Quantifying disturbance effects on vegetation carbon pools in mountain forests based on historical data. Reg Environ Change 9: 121-130.

GINZLER C, BRÄNDLI UB, HÄGELI M (2011) Waldflächenentwicklung der letzten 120 Jahre in der Schweiz. Schweiz Z Forstwes 162: 337-343. doi: 10.3188/szf.2011.0337

HANEWINKEL M, ALBRECHT A, SCHMIDT M (2015) Können Windwurfschäden vermindert werden? Eine Analyse von Einflussgrössen. Schweiz Z Forstwes 166: 118-128. doi: 10.3188/ szf.2015.0118

KURZ D, ALVETEG M, SVERDRUP H (1998) Acidification of Swiss forest soils. Development of a regional dynamic assessment. Bern:. Swiss Agency for the environment, Forests and Landscape (SAEFL), Environmental Documentation 89. 115 p. 
LANDOLT E (1861) Witterungserscheinungen im Jahr 1860 und deren Einfluss auf die Vegetation und die Waldarbeiten. Schweiz Z Forstwes 11: 29-33.

LANDOLT E (1862) Bericht an den hohen schweizerischen Bundesrath über die Untersuchung der schweiz. Hochgebirgswaldungen, vorgenommen in den Jahren 1858, 1859 und 1860. Bern: Fiala. 367 p.

MITCHELL SJ (2013) Wind as a natural disturbance agent in forests: a synthesis. Forestry 86: 147-157

MÜNCHNER RÜCK (2001) Winterstürme in Europa (II): Schadenanalyse 1999 - Schadenpotenziale. München: Münchner Rückversicherungs-Gesellschaft. $74 \mathrm{p}$.

OTT E (1972) Erhebungen über den gegenwärtigen Zustand des Schweizer Waldes als Grundlage waldbaulicher Zielsetzungen. Teil A. Mitt Schweiz Anstalt forstl Vers.wes 48: 1-193.

PFISTER C (1998) Raum-zeitliche Rekonstruktion von Witterungsanomalien und Naturkatastrophen 1496-1995. Zürich: VDF. $140 \mathrm{p}$.

PFISTER C (1999) Wetternachhersage - 500 Jahre Klimavariationen und Naturkatastrophen (1496-1995). Bern: Haupt. 304 p.

PFISTER C (2009) Die «Katastrophenlücke» des 20. Jahrhunderts und der Verlust traditionalen Risikobewusstseins. Gaia 1: 239_ 246.
SCHELHAAS MJ, NABUURS GJ, SCHUCK A (2003) Natural disturbances in the European forests in the $19^{\text {th }}$ and $20^{\text {th }}$ centuries. Glob Chang Biol 9: 1620-1633.

SEIDL R, RAMMER W, BLENNOW K (2014A) Simulating wind disturbance impacts on forest landscapes: Tree-level heterogeneity matters. Environ Modell Softw 51: 1-11.

SEIDL R, SCHELHAAS MJ, RAMMER W, VERKERK PJ (2014B) Increasing forest disturbances in Europe and their impact on carbon storage. Nat Clim Chang 4: 806-810.

USBECK T, WOHLGEMUTH T, PFISTER C, VOLZ R, BENISTON M (2010A) Wind speed measurements and forest damage in Canton Zurich (Central Europe) from 1891 to winter 2007. Int J Climat 30: 347-358.

USBECK T, WOHLGEMUTH T, DOBBERTIN M, PFISTER C, BÜRGI A ET AL (2010B) Increasing storm damage to forests in Switzerland from 1858 to 2007. Agric For Meteorol 150: 47-55.

WOHLGEMUTH T, CONEDERA M, KUPFERSCHMID ALBISETTI A, MOSER B, USBECK T ET AL (2008) Effekte des Klimawandels auf Windwurf, Waldbrand und Walddynamik im Schweizer Wald. Schweiz Z Forstwes 159: 336-343. doi: 10.3188/ szf.2008.0336

WSL, BUWAL, EDITORS (2001) Lothar. Der Orkan 1999. Ereignis analyse. Birmensdorf: Eidgenöss Forsch.anstalt WSL. 365 p.

\section{Tempêtes hivernales dans les forêts suisses de 1865 à 2014}

Les tempêtes hivernales causent les dégâts les plus conséquents à la forêt suisse. Il a été étudié, pour le présent article, comment se sont développés les dégâts aux forêts causés par des tempêtes hivernales en Suisse et dans les cantons entre 1865 et 2014 en fonction de la force du vent, du matériel sur pied et en rapport avec la surface forestière. Durant la période considérée, il y a eu 26 tempêtes hivernales avec au minimum $70000 \mathrm{~m}^{3}$ de chablis. Les dégâts présentent une forte variabilité aussi bien absolue $\left(\mathrm{m}^{3}\right)$, relative $\left(\mathrm{m}^{3} / \mathrm{ha}\right)$ qu'en pourcentage du matériel sur pied (\%). Au cours des 150 dernières années, les cantons de Nidwald, Fribourg, Argovie, Zurich et Zoug ont, en moyenne, été les plus fortement touchés avec des dégâts de $2.2 \mathrm{~m}^{3} /$ ha (Zurich) à $3.1 \mathrm{~m}^{3} / \mathrm{ha}$ (Nidwald) par évènement. Le passage du nouvel millénaire a enregistré les plus forts dégâts, les plus forts volumes sur pied ainsi que les vents les plus violents.

\section{Forest damages from winter storms in Switzerland from 1865 to 2014}

Winter storms cause the most catastrophic damages in Swiss forests. The present article analyses how these storm damages correspond with wind gust speed, growing stock and forest area, in regard to the whole country and individual cantons, and from 1865 to 2014. During the study period, 26 storm events each totalling a volume of at least $70,000 \mathrm{~m}^{3}$ damaged wood were registered. Winter storm damages were highly variable regarding absolute numbers (volume) and portions per area ( $\mathrm{m}^{3}$ per ha) and per growing stock (\%). In the past 150 years, the cantons Nidwalden, Freiburg, Aargau, Zurich and Zug were hit most often by storm events, with damages ranging per event in average from $2.2 \mathrm{~m}^{3}$ per ha ( $\mathrm{Zu}$ rich) to $3.1 \mathrm{~m}^{3}$ per ha (Nidwalden). At the turn of the millennium, not only the greatest damages occurred but also growing stock peaked as well did the wind gust speeds. 